\title{
Le dernier verre - dernières nouvelles du programme médical de prévention d'une consommation d'alcool à risque
}

\author{
B. Stoll
}

Deutsch erschienen

in Nr. 35/2005

Correspondance:

Dr Beat Stoll, MPH

Institut de médecine sociale et préventive

Centre Médical Universitaire

CH-1211 Genève 4

E-mail: beat.stoll@imsp.unige.ch
En l'an 2000, nous avons lancé le projet partiel «médecins» et relevé le défi, jusqu'au milieu de l'année 2004, de collaborer à la campagne nationale de prévention de l'alcoolisme organisé par l'Office fédéral de la santé publique intitulé «Ça débouche sur quoi?». Le but consistait tout d'abord à sensibiliser les médecins, mais aussi les autres professionnels de la santé afin de reconnaître le plus rapidement possible les patients présentant une consommation d'alcool à risque et de leur offrir un instrument de conseil grâce aux interventions brèves.

Nous avons eu la chance de nous engager dans un projet sur plusieurs années, marqué principalement par l'enthousiasme des participants et par le fait que le programme s'est modifié, a évolué et gagné en ouverture. Les difficultés d'application auxquelles nous avons été confrontés sont inhérentes à ce type de projet, mais elles sont formatrices et ce, même si elles sont inattendues.

Au premier abord, il nous semblait évident de pouvoir enfin, en ce troisième millénaire, parler de prévention au cabinet médical. Aborder la question de l'alcool ne fut toutefois pas si simple. En effet, quiconque souhaite aborder la question de la consommation d'alcool à risque et ses conséquences avec les médecins doit s'attendre à ce que le public se pose une foule de questions sur les exigences de traitement des patients alcoolodépendants. Dans ce contexte, parler de prévention en obtenant l'attention voulue relève du défi. La création d'un atelier interactif qui permettrait aux participants de confronter leurs expériences s'est avéré indispensable. Les interventions brèves nous ont fourni un prétexte idéal pour remettre fondamentalement en question la manière de conduire les entretiens avec les patients. L'alcool est un sujet idéal, car tous les participants y sont sensibles, ce qui leur permet de détecter rapidement de part et d'autre les comportements offensants (tant de la part du médecin que du patient). La réalisation de jeux de rôle en petits groupes nous ont ainsi permis de nous rapprocher de la réalité. En outre et grâce à la familiarisation avec les interventions brèves, il a également été possible de faire passer un tout autre message, à savoir que nous ne devons pas chercher à convaincre nos patients par de longs discours, mais à les aider à trouver le moyen de réduire (ou non) leur consommation à risque d'alcool.

En plus de quatre ans, nous avons organisé quelque 70 manifestations, principalement des ateliers comprenant en moyenne une douzaine de participants. En outre, nous avons été invités à présenter des exposés dans la plupart des grands congrès des sociétés de médecine de premier recours et avons également organisé des sessions de formation continue dans divers hôpitaux régionaux. Plus de mille médecins et autres professionnels de la santé ont été sensibilisés à la question de la consommation d'alcool à risque. Nous avons aussi régulièrement publié de brefs articles dans le bulletin d'information «Flaschenpost» pour étayer nos propos et mettre en lumière d'autres aspects du programme.

Les trois grandes manifestations organisées en collaboration avec nos institutions et organisations partenaires ont représenté sans conteste le point culminant de notre travail. Elles ont également reflété l'orientation du programme. Pour ce qui est du lancement du projet sur le plan universitaire, deux groupes d'experts se sont réunis durant deux jours à Lausanne en septembre 2000, lors du symposium Widmer intitulé «Implementation of early interventions for hazardous drinkers in primary care» organisé en collaboration avec le Dr Jean-Bernard Daeppen, directeur du Centre de traitement en alcoologie (CTA) du CHUV de Lausanne, pour se consacrer au thème de l'alcool et de l'intervention brève. Le premier groupe d'experts comprenait des spécialistes du monde entier qui analysent scientifiquement depuis plusieurs années la question de l'intervention brève lors de consommation d'alcool à risque. Le deuxième groupe était quant à lui composé d'experts en médecine générale exerçant en pratique privée en Suisse. Le premier jour du symposium, les médecins suisses ont pu tirer profit des expériences de leurs confrères étrangers, alors que le deuxième jour ces derniers 
ont pu se familiariser avec la pratique suisse en matière de prévention de l'alcoolisme. Les deux groupes sont repartis très satisfaits.

La deuxième manifestation d'envergure était le congrès organisé en 2002 à Berne par la Société suisse d'alcoologie. Des circonstances plus ou moins heureuses nous ont étonnamment permis d'organiser dans les plus brefs délais un congrès consacré aux interventions brèves dans la prévention de la consommation d'alcool à risque. Ainsi, il nous a été possible de réunir à Berne plus de 110 professionnels de la santé provenant de toute la Suisse, lesquels ont pu s'essayer aux différents aspects des interventions brèves. Cette manifestation nous a permis d'élargir notre public-cible, car la prévention de l'alcoolisme ne saurait se limiter aux seuls médecins. C'est d'une importance non négligeable pour la suite de l'expérience.

Pour couronner le tout, la possibilité nous a été offerte d'organiser, en collaboration avec le Centre de coordination et de soutien pour les groupes d'entraide en Suisse (KOSCH), la Société suisse de médecine générale et le programme de prévention du tabagisme et du suicide de la FMH, un séminaire consacré à la relation médecinpatient destiné aux patients, à leurs proches et au corps médical. L'idée était que toutes les parties prennent la parole et réfléchissent ensemble sur les difficultés et les chances de la relation médecin-patients-proches. Symboliquement, cette manifestation était placée sous le signe de la mutation du rapport médecinpatient, qui nous tient à cœur dans notre programme. La différenciation expert/personne inexpérimentée devrait être prise en compte par les deux parties. A cela s'ajoute que l'abord plutôt vertical de la prévention de l'alcoolisme a aussi ses limites (en particulier lorsqu'on parle de consommation à risque). L'intervention brève est un instrument qui comporte plusieurs possibilités d'application.

Qu'en est-il du projet partiel Quo-vadis de la campagne «Ça débouche sur quoi?». En Suisse romande, le projet est solidement ancré. Le Centre de Traitement en Alcoologie (CTA) du CHUV a développé un matériel didactique intéressant à ce propos et le propose à diverses divisions hospitalières. Par le biais de notre programme dans le canton de Genève, nous avons pu former plusieurs représentants de diverses professions médicales à même de former ensuite leurs confrères. Le service social poursuit ce programme de manière autonome et prévoit de motiver toutes les personnes actives dans les soins à domicile ou dans le domaine social à s'engager dans la prévention de l'alcoolisme. D'autres villes et cantons ne devraient pas tarder à suivre cet exemple, car ce n'est qu'ainsi qu'il sera possible de reconduire le programme de prévention l'année prochaine et de lui fournir une assise plus étendue. Il y a peu, un groupe d'experts de l'OMS a estimé que l'intervention brève représentait la méthode de prévention de l'alcoolisme la plus efficace [1] (cf. aussi [2]). Malheureusement, la Suisse manque encore de volonté politique confédérale pour poursuivre dans cette voie et le peu de moyens financiers restants sont toujours investis dans des campagnes d'affichage déconcertantes et peu coordonnées qui, d'un point de vue scientifique, n'ont qu'une faible, voire aucune incidence.

En conclusion, nous levons nous aussi symboliquement notre dernier verre au manuel d'auto-apprentissage sur «Les interventions brèves pour les consommateurs d'alcool à risque» publié aux éditions EMH. Ce manuel est le fruit du travail de notre groupe d'experts*, qui s'est créé et consolidé au cours des presque 5 années de travail commun avec le soutien de l'Office fédéral de la santé publique et de l'Institut suisse de prévention de l'alcoolisme et autres toxicomanies à Lausanne. En quelque 40 pages, le lecteur pourra se familiariser progressivement avec le sujet, il y trouvera des conseils pratiques et certains concepts, ainsi qu'un résumé de tous les travaux scientifiques importants dans ce domaine. Le public-cible auquel s'adresse le manuel montre également les limites d'un tel projet: en règle générale, il n'aboutit que dans les mains de confrères déjà convaincus. Néanmoins, si ce manuel incite à la réflexion, nous aurons tout de même fait un grand pas en avant. Pour terminer, relevons qu'aucune brochure ne saurait remplacer les précieux échanges en atelier interactif. Par conséquent, nous vous invitons à y participer et trinquons au succès de cette entreprise.

Les brochures peuvent être commandées auprès de la FMH au prix de revient de Fr. 10.- (Mme B. Weil, service de prévention de la FMH, Elfenstrasse 18, 3000 Berne 15, e-mail: barbara.weil@ hin.ch).

\section{Références}

1 WHO. Alcohol is not an ordinary drug. Geneva: WHO; 2004.

2 Babor TF, Caetano R, Casswell S, et al. Alcohol: No Ordinary Commodity - Research and Public Policy. Oxford, London: Oxford University Press; 2003.
A. M. Keller; P. Loeb, Bâle;
L. Matter, Loèche; M. Peltenburg,

Hinwil; Prof. M. Sieber, Zurich. 